\title{
Very high energy gamma-ray observations of the Galactic Plane with the CANGAROO-III telescopes
}

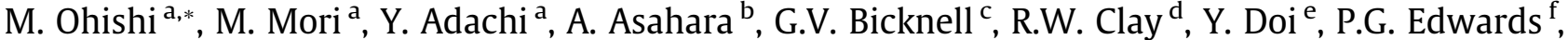 \\ R. Enomoto ${ }^{\text {, }}$, S. Gunji ${ }^{\text {e, S. Hara }}{ }^{\mathrm{g}}$, T. Hara ${ }^{\mathrm{h}}$, T. Hattori ${ }^{\mathrm{i}}$, Sei. Hayashi ${ }^{\mathrm{j}}$, Y. Higashi ${ }^{\mathrm{b}}$, Y. Hirai ${ }^{\mathrm{k}}$, K. Inoue ${ }^{\mathrm{e}}$, \\ C. Itoh $^{\text {g}}$, S. Kabuki ${ }^{\text {b }}$, F. Kajino ${ }^{j}$, H. Katagiri ${ }^{1}$, A. Kawachi ${ }^{i}$, T. Kifune ${ }^{a}$, R. Kiuchi ${ }^{a}$, H. Kubo ${ }^{b}$, T. Kurihara ${ }^{i}$, \\ R. Kurosaka ${ }^{a}$, J. Kushida ${ }^{i}$, Y. Matsubara ${ }^{m}$, Y. Miyashita ${ }^{i}$, T. Mizukami ${ }^{\text {b }}$, R. Mizuniwa ${ }^{i}$, H. Muraishi $^{\text {n }}$, \\ Y. Muraki ${ }^{\mathrm{m}}$, T. Naito $^{\mathrm{h}}$, T. Nakamori ${ }^{\mathrm{b}}, \mathrm{S}$. Nakano ${ }^{\mathrm{b}}$, T. Nakase $^{\mathrm{i}}$, D. Nishida ${ }^{\mathrm{b}}$, K. Nishijima ${ }^{\mathrm{i}}$, N. Sakamoto ${ }^{\mathrm{e}}$,

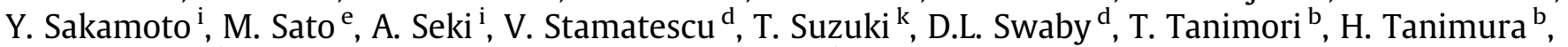 \\ G.J. Thornton ${ }^{\mathrm{d}}$, F. Tokanai ${ }^{\mathrm{e}}$, K. Tsuchiya ${ }^{\mathrm{o}}$, S. Watanabe ${ }^{\mathrm{b}}$, Y. Yamada $^{\mathrm{j}}$, T. Yamaoka $^{\mathrm{j}}$, E. Yamazaki $^{\mathrm{i}}$, \\ S. Yanagita ${ }^{k}$, T. Yoshida $^{k}$, T. Yoshikoshi $^{\text {a }}$, Y. Yukawa $^{\text {a }}$
}

a Institute for Cosmic Ray Research, University of Tokyo, Kashiwa, Chiba 277-8582, Japan

${ }^{\mathrm{b}}$ Department of Physics, Graduate School of Science, Kyoto University, Sakyo-ku, Kyoto 606-8502, Japan

${ }^{\mathrm{c}}$ Research School of Astronomy and Astrophysics, Australian National University, ACT 2611, Australia

${ }^{\mathrm{d}}$ Department of Physics and Mathematical Physics, University of Adelaide, SA 5005, Australia

e Department of Physics, Yamagata University, Yamagata, Yamagata 990-8560, Japan

${ }^{\mathrm{f}}$ Paul Wild Observatory, CSIRO Australia Telescope National Facility, Narrabri, NSW 2390, Australia

${ }^{\mathrm{g}}$ Ibaraki Prefectural University of Health Sciences, Ami, Ibaraki 300-0394, Japan

${ }^{\mathrm{h}}$ Faculty of Management Information, Yamanashi Gakuin University, Kofu, Yamanashi 400-8575, Japan

i Department of Physics, Tokai University, Hiratsuka, Kanagawa 259-1292, Japan

${ }^{\mathrm{j}}$ Department of Physics, Konan University, Kobe, Hyogo 658-8501, Japan

${ }^{\mathrm{k}}$ Faculty of Science, Ibaraki University, Mito, Ibaraki 310-8512, Japan

${ }^{1}$ Department of Physical Science, Hiroshima University, Higashi-Hiroshima, Hiroshima 739-8526, Japan

${ }^{\mathrm{m}}$ Solar-Terrestrial Environment Laboratory, Nagoya University, Nagoya, Aichi 464-8602, Japan

${ }^{\mathrm{n}}$ School of Allied Health Sciences, Kitasato University, Sagamihara, Kanagawa 228-8555, Japan

${ }^{\circ}$ National Research Institute of Police Science, Kashiwa, Chiba 277-0882, Japan

\section{A R T I C L E I N F O}

\section{Article history:}

Received 27 February 2008

Received in revised form 22 May 2008

Accepted 12 June 2008

Available online 3 July 2008

\section{PACS:}

95.85.Pw

98.35. $-\mathrm{a}$

Keywords:

Gamma-rays: observations

Milky way galaxy

\begin{abstract}
A B S T R A C T
In 2004, we searched for very high energy (VHE) gamma-ray emission from the Galactic Plane using the CANGAROO-III stereoscopic observation system. Two different longitude regions $\left(\ell=-19^{\circ} .5\right.$ and $\ell=+13^{\circ} .0$ ) on the Galactic Plane were observed during July and August 2004. We analyzed events that triggered three telescopes aiming to measure the diffuse emission component. No significant signal associated with the Galactic Plane was found from either of the regions. Assuming that the gamma-ray spectrum is described by a single power-law for energies ranging between a few $\mathrm{GeV}$ and $\mathrm{TeV}$, lower limits of the power-law spectral indices were found to be 2.2 for both of the regions with a $99.9 \%$ confidence level. This result is consistent with the other VHE measurements and constrains a hypothesis in which a very hard $(\sim 2.0)$ cosmic ray electron spectrum was introduced to explain the EGRET GeV anomaly.
\end{abstract}

(c) 2008 Elsevier B.V. All rights reserved.

\section{Introduction}

The Galactic Plane is known to be a very bright source of gamma-rays in the GeV energy band. Its gamma-ray spatial profile and spectrum across the entire Galactic Plane were measured by satel-

\footnotetext{
* Corresponding author. Tel.: +81 47136 5144; fax: +81 471363133.

E-mail address: ohishi@icrr.u-tokyo.ac.jp (M. Ohishi).
}

lite experiments such as SAS-II, COS-B and CGRO. Among these, the EGRET detector on the CGRO [1] was sensitive to GeV gamma-rays, and the observations using this detector provided the detailed spatial and spectral characteristic features of gamma-ray emission from the Galactic Plane up to $30 \mathrm{GeV}$.

Gamma-rays from the Galactic Plane are believed to originate from the interaction between galactic cosmic-ray protons/electrons and target matter/photons concentrated in the plane. Since 
the cross sections of gamma-ray production processes (the decay of neutral pions generated in proton-proton collision, bremsstrahlung and inverse Compton scattering by high energy electrons) are known with satisfactory accuracy, both the spatial and spectral characteristic features of gamma-ray emission from the Galactic Plane can be estimated if following parameters are known: (1) density distribution of the target matter, (2) cosmic ray proton/ electron spectrum, and (3) cosmic ray spatial intensity distribution. Some of these parameters are obtained by radio observations or direct cosmic-ray measurement near the Earth.

In this context, some authors constructed gamma-ray emission models for the Galactic Plane, and among these, the model by Bertsch [2] was in good agreement with the results obtained from SAS-II and COS-B. Based on this model, Hunter et al. [3] constructed an improved model that incorporated the most current radio observation results to compare with the EGRET GeV measurement.

This "EGRET diffuse emission model" showed good agreement with the EGRET measurement of spatial and spectral features in the sub-GeV region. However in higher energy regions above $1 \mathrm{GeV}$, the measured flux was $60 \%$ greater than that of the model, despite the fact that the spatial characteristic features were still in agreement. Furthermore, the power-law spectral index measured between 1 and $30 \mathrm{GeV}$ was harder and had an average value of 2.45 , which differed from the value expected from the cosmicray proton spectrum measured on the Earth $(\sim 2.7)$. This discrepancy is known as the "GeV anomaly", and many hypotheses have been proposed to explain it.

From technical viewpoint, one of the most probable causes for this discrepancy is that there might be systematic error in the estimated gamma-ray acceptance of the EGRET detector, particularly in the high energy region. A previous study [3] revealed that many of the detected point sources had an energy spectrum described by a single power-law without a break around $1 \mathrm{GeV}$, which did not support the possibility that acceptance estimation errors occur only in the high energy $(E>1 \mathrm{GeV})$ band. Recently Stecker et al. [4] pointed out that after re-examining of high quality events that were selected carefully, the EGRET "GeV anomaly" could be due to a systematic error in the estimate of the detector's acceptance at energies above $1 \mathrm{GeV}$. They supported their claim by presenting indirect evidence by re-analyzing known EGRET sources provided in the paper; However, direct experimental evidence is currently unavailable.

From an astrophysical viewpoint, one of the possible explanations for the origin of the GeV anomaly is that the cosmic-ray spectrum adopted in the model was not as suitable as the Galactic average. A harder proton/electron injection spectrum produces more intense gamma-ray emission in the $\mathrm{GeV}$ region, leading to the $\mathrm{GeV}$ excess. Regarding the electron spectrum, a spectral index of 2.4 was used for the EGRET diffuse model which fits the local electron flux up to $1 \mathrm{TeV}$. Pohl et al. [5] proposed a very hard cosmic-ray electron injection (spectral index of $\sim 2.0$ ) supported by radio observations of individual supernova remnants. In their hypothesis, the inverse Compton component is enhanced in the higher energy region, and is dominant at a few hundred $\mathrm{GeV}$ with a gamma-ray spectral index of $\sim 1.85$. Regarding the possibility of a hard proton spectrum, Mori [6] showed that a spectral index of 2.45 provides a better fit to the EGRET measurement in the GeV region; however, the gamma-ray flux in the $\mathrm{MeV}$ region is over-predicted even after considering the uncertainty in the kinetic energy distribution of pions of a few $\mathrm{GeV}$ in the study. Kamae et al. [7] performed a detailed Monte Carlo simulation study on a $p p \rightarrow \pi^{0}$ process which incorporated a diffractive process and Feynman scaling violation, and showed that if the cosmic-ray proton spectrum is slightly $(\sim 0.2)$ harder than the local measured $(\sim 2.7)$ one, the $\mathrm{GeV}$ excess can be completely explained. Strong, Moskalenko and
Reimer [8] devised an optimized model to fit the EGRET data by varying both the proton and the electron spectra. The simplest test for these hypotheses is to measure the spectral shape more precisely at higher energy, i.e. in the TeV range, by an independent detector covering the entire Galactic Plane. Therefore observations performed using an imaging atmospheric Cherenkov telescope (IACT hereafter) in the $\mathrm{TeV}$ region can constrain these hypotheses.

Ground-based observations of VHE gamma-rays using IACTs developed rapidly after the introduction of the imaging method and detection of the Crab nebula with the Whipple telescope $([9,10])$ in the late 1980 s. Along with improvement of the detectors, the number of targets detected in VHE band by this technique has been increasing and reached 71 in 2007 [11]. At present, stereoscopic observation systems with $10 \mathrm{~m}$-class reflectors, such as CANGAROO-III [12], H.E.S.S. [13], and VERITAS [14] and single large (17 m-diameter) reflector system MAGIC [15], are exploring the VHE universe with an angular resolution of a few arcmin. Among past IACTs, Whipple and HEGRA (the predecessor of VERITAS and H.E.S.S.) groups made observations of the Galactic Plane inspired by [3]. However, they did not find a significant gamma-ray signal $([16,17])$. The results of the past VHE observations are summarized in Table 1. These results constrained the hard electron spectrum hypothesis [5] mentioned above. Among present generation experiments, the H.E.S.S. telescope conducted a Galactic Plane survey [18] in 2004-2005 at energies ranging from 0.2 to $10 \mathrm{TeV}$, and reported that they detected diffuse gamma-ray emission from the Galactic Plane, near the Galactic Center, after subtracting the point source emission component [19]. The spectral index of the gammaray emission was reported to be $2.29 \pm 0.07_{\text {stat }} \pm 0.02_{\text {sys }}$ for $\left(|\ell|<0^{\circ} .8\right.$ and $|b|<0^{\circ} .3$ ). In addition to IACTs, Milagro (water Cherenkov detector array, [20]) claim the detection of a gamma-ray signal associated with the Galactic Plane ([21]) from the Cygnus Region with a spectral index between 2.3 and 2.4 for the $\left(\ell=65^{\circ}-85^{\circ},|b|<3^{\circ}\right)$ region.

The CANGAROO-III stereoscopic observation system is located in the southern hemisphere $\left(136^{\circ} .8 \mathrm{E}, 31^{\circ} .1 \mathrm{~S}\right)$, ideally suited for observations toward the galactic center. Motivated by the discussions about EGRET GeV anomaly problem mentioned above, the Galactic Plane was observed by the CANGAROO-III telescope in 2004 , and the results of this analysis are reported.

\section{Observations}

We observed the Galactic Plane using the CANGAROO-III stereoscopic system in July and August, 2004. The relevant details of the CANGAROO-III system and its detectors were described in [22-25], and we provide a brief overview here. The system consists of four $10 \mathrm{~m}$-diameter telescopes arranged in a diamond shape with 100 $\mathrm{m}$ sides. The reflector is a tessellated parabola with 114 spherical

Table 1

Summary of results of the VHE observation of the Galactic Plane

\begin{tabular}{llllc}
\hline Group & Detector & $E_{\text {th }}$ & Observed point & Index \\
\hline Whipple [16] & IACT (single) & 0.5 & $(\ell, b)=\left(+40^{\circ} .0,0^{\circ} .0\right)$ & $>2.31$ \\
HEGRA [17] & IACT (stereo) & 1 & $\ell=37^{\circ}-43^{\circ},|b|<5^{\circ}$ & $>2.5$ \\
Milagro [21] & Water Cherenkov & $12^{\mathrm{a}}$ & $\ell=65^{\circ}-85^{\circ},|b|<3^{\circ}$ & $2.3-2.4$ \\
Tibet-III [33] & AS array & 3 & $\ell=20^{\circ}-55^{\circ},|b|<2^{\circ}$ & $>2.0^{\mathrm{b}}$ \\
H.E.S.S. [19] & IACT (stereo) & 0.2 & $|l|<0^{\circ} .8,|b|<2^{\circ}$ & $2.3^{\mathrm{c}}$ \\
CANGAROO-III & IACT (stereo) & 0.6 & $(\ell, b)=\left(-19^{\circ} .5,0^{\circ} .0\right)$ & $>2.2$ \\
CANGAROO-III & IACT (stereo) & 0.6 & $(\ell, b)=\left(+13^{\circ} .0,0^{\circ} .0\right)$ & $>2.2$ \\
\hline
\end{tabular}

Unit of the energy threshold is TeV.

a This figure is as a median energy.

b Tibet group reported positive signal detection from the Cygnus region later in [34], but they did not present value of the gamma-ray spectral index considering low gamma/hadron separation efficiency of their detector.

c $2.29 \pm 0.07_{\text {stat }} \pm 0.02_{\text {syst }}$, to be precise. 
mirror segments and a total surface area of $54 \mathrm{~m}^{2}$. The imaging camera design for the three newer telescopes are identical and consists of 427 close-packed photo multiplier tube (PMT) pixels. Each telescope's field of view is $4^{\circ} .0$, with a pixel-size of $0^{\circ} .168$. Because the field of view of the oldest telescope is smaller $\left(3^{\circ} .0\right)$ than that of the other three telescopes, the data obtained using this telescope were not used in the stereo analysis. Analog signals from the PMT pixels are amplified and fed into the electronic modules, where the signal amplitude/arrival time is digitized by an analogue-to-digital converter (ADC) and a time-to-digital converter (TDC). The output of these electronic modules is recorded with a GPS time-stamp, which has $1 \mu$ s accuracy. In 2004, the event coincidence between the telescopes was reconstructed offline using a software applying this GPS time information. The online stereo coincidence trigger system was introduced later in 2005. The obtained raw ADC/TDC data was converted into a photo-electron/ns unit using daily LED (Light Emitting Diode) flash calibration.

Since the Galactic Plane is large with respect to the field of view of the CANGAROO-III camera, we decided to observe a few points on the Galactic Plane and define them as target points rather than making a low sensitivity scan over a wide area along the Galactic longitude. The target points were selected to satisfy the following conditions:

(1) They must be coincident with local emission maxima of the EGRET diffuse model. (see Fig. 1)

(2) The minimum observation zenith angle at the CANGAROOIII site should be less than $20^{\circ}$.

(3) No bright ( $<4.0$ magnitude) star is located in the field of view.

(4) No known TeV gamma-ray sources can be inside the field of view.

According to the above conditions, two points, $(\ell, b)=\left(-19^{\circ} .5\right.$, $\left.0^{\circ} .0\right)$ and $\left(+13^{\circ} .0,0^{\circ} .0\right)$, were selected. After this observation the H.E.S.S. group found a TeV point source, HESS J1813-178, in the field of view of the $\ell=+13^{\circ} .0$ region, violating the fourth condition. The $\ell=-19^{\circ} .5$ region was observed in the scanning mode in the Galactic latitude direction with a scan point interval of $3^{\circ} .0$. Each scan point was observed for $15 \mathrm{~min}$, and then the tracking point was switched to neighboring point (see Fig. 1). Regions at $|b|=3^{\circ} .0$ were used as a "background" with respect to the on-plane region in the analysis of the diffuse emission from the disk. For the $\ell=+13^{\circ} .0$ region, a conventional long ON/OFF observation mode (the off-source path in the celestial coordinate was selected to be the same as that of the on-sources) was adopted, since the variation of the optical brightness in the scanning path was rather large, and the trigger rate fluctuation was problematic without an online coincidence trigger system. All the selected OFF-source positions were well away from the Galactic Plane. This study only considers 3-fold coincident events, i.e. events triggered by all of the three newer telescopes. We utilized as much image information as we had, in order to obtain
Table 2

Total time and zenith angle range of the Galactic Plane observation by the CANGAROO-III in 2004

\begin{tabular}{llll}
\hline Target point & & Observation time $(\mathrm{h})$ & Zenith angle \\
\hline$(\ell, b)=\left(-19^{\circ} .5,0^{\circ} .0\right)$ & $(\mathrm{ON})$ & 8.0 & $12^{\circ}-49^{\circ}$ \\
$(\ell, b)=\left(-19^{\circ} .5,+3^{\circ} .0\right)$ & $(\mathrm{OFF})$ & 4.1 & \\
$(\ell, b)=\left(-19^{\circ} .5,-3^{\circ} .0\right)$ & $(\mathrm{OFF})$ & 4.3 & $13^{\circ}-40^{\circ}$ \\
$(\ell, b)=\left(+13^{\circ} .0,0^{\circ} .0\right)$ & $(\mathrm{ON})$ & 5.9 & \\
& $(\mathrm{OFF})$ & 6.1 &
\end{tabular}

better proton/gamma separation power in the diffuse source analysis. The net length of the time used in the analysis are summarized in Table $2 ; 8.0$ hours for the $\ell=19^{\circ} .5$ and 5.9 hours for the $\ell=+13^{\circ} .0$ region. These observations were made at zenith angles less than $50^{\circ}$, and the energy threshold averaged over all observation zenith angles was estimated to be $600 \mathrm{GeV}$,

\section{Data analysis}

The analysis in this study follows [26-28]. We briefly outline the procedure here.

To extract the shower from the noise contaminated images, triggered pixels with a photon intensity of more than 5 p.e. and whose photon arrival timing was within a \pm 30 ns time-window were selected, and the other PMT pixels were rejected from the analysis. Clusters of at least five adjacent triggered pixels were required after these image cleaning. Typical event rate after this procedure was $7.0 \mathrm{~Hz}$ for good weather conditions. Next, shower images that were artificially truncated due to the finite field of the imaging camera were rejected if any of the brightest 15 pixels was is in the outermost layer of the imaging camera. This method is a slightly improved version of the method described by [27] for the purpose of enhancing high energy gamma-ray acceptance.

To determine the shower arrival direction, we used both information about the shower axis direction and the distance relation between the target position and the image centroid. The most suitable arrival direction was then determined by conducting a grid search. (All the details about this method are described in [28].) This procedure provides a better angular resolution, particularly for large zenith angles. Assuming a point-like source, $27 \%$ of gamma-ray events is included within a $0^{\circ} .1$ radius using this reconstruction method.

We adopted Fisher Discriminant (FD hereafter) fit method to extract gamma-ray like events from the background, and detail about this analysis is described in [26]. In the MC dataset used in the FD fit procedure, we assumed a single power-law spectrum with index of -2.4. As for the spatial extent, we referred to the EGRET emission profile for $E>1 \mathrm{GeV}$ events (see Fig. 2) for which the width of the signal peak associated with the Galactic Plane was $1^{\circ} .1$ and $0^{\circ} .8$ for $\ell=-19^{\circ} .5$ and $\ell=+13^{\circ} .0$ in Fig. 2. Point spread function of EGRET at $5 \mathrm{GeV}$ is $0^{\circ} .5$ (as $67 \%$ containment radius [30]) and the obtained extent is larger than this PSF. In our MC simulation, the emission

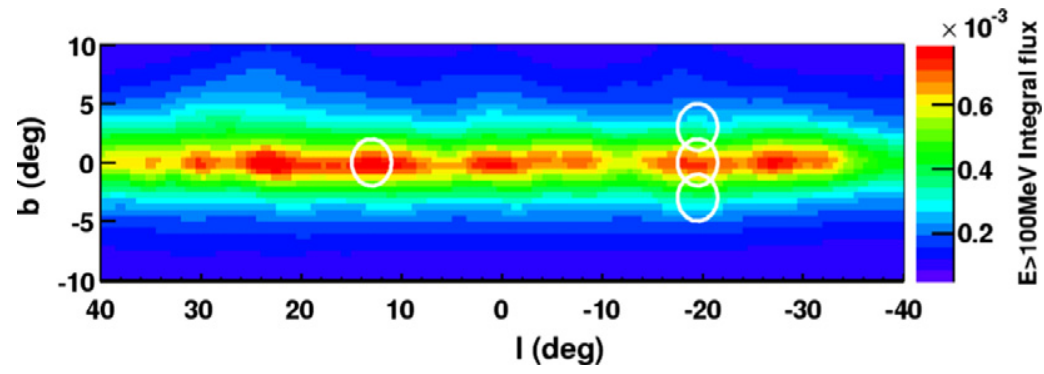

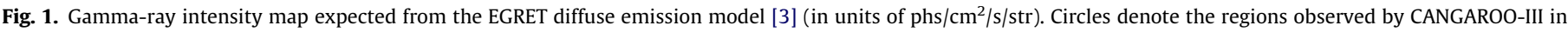
2004. 


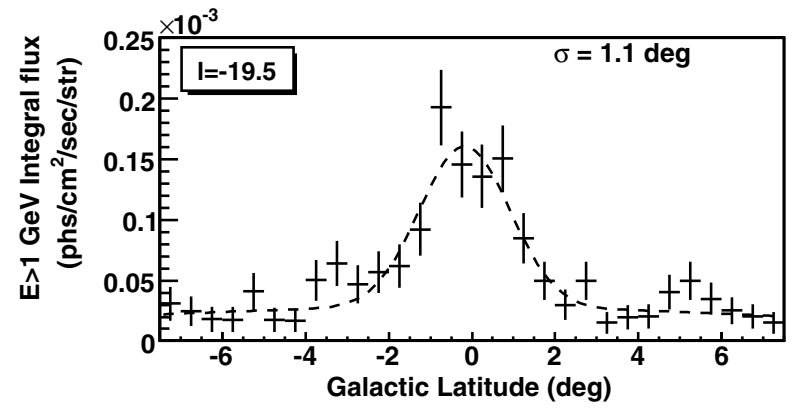

(a) $\ell=-19^{\circ} .5$

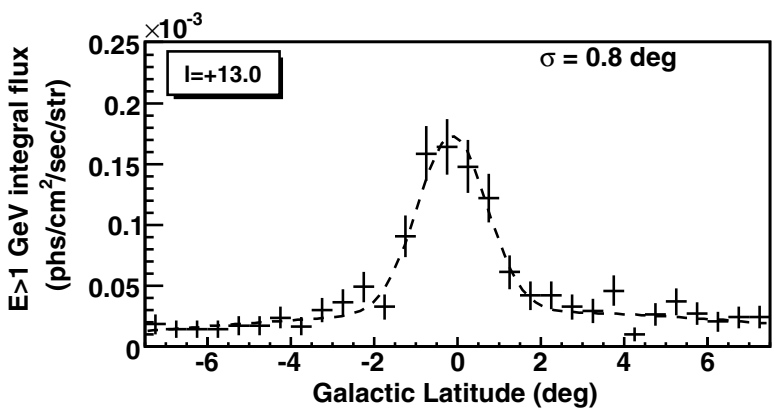

(b) $\ell=+13^{\circ} .0$

Fig. 2. EGRET flux intensity profile along the Galactic latitude for $E>1 \mathrm{GeV}$ events [29]. Integration range in the longitude is (a) $-20^{\circ} .0$ to $-19^{\circ} .0$ and (b) $12^{\circ} .5-13^{\circ} .5$. Vertical bars correspond to 1 sigma statistical error. Broken lines show fitting results with double Gaussian function and obtained sigma values for the center peaks are shown the figures.

profile was simplified to be uniform within circles with radii of $1^{\circ} .0$ and $0^{\circ} .8$.

In the analysis of an extended source whose emission size is comparable or larger compared with the size of the imaging camera, obtained excess count value is sensitive to the accuracy of background level estimation. Unlike a point-source analysis where the background level can be defined within the same field of view, we need independent OFF-source data and its observation/analysis condition should be identical to the ON-source. In our analysis a small difference of azimuth/elevation angle distribution between ON/OFF-source data which remained after the image cleaning procedure was corrected by applying an appropriate weight to OFFsource events. Though uncertainty of ON/OFF normalization factor is important in the background level estimation in the conventional cut and subtract method, our fit analysis method uses only shape information and this ON/OFF normalization factor is set free. Shape parameters and event acceptance have dependence on the image position on the camera and this effect should be considered when we calculate spatial distribution of gamma-ray flux. In the derivation of the 2-dimensional/1-dimensional spatial distribution plot, we divided ON and OFF-source data into subsets according to their arrival direction and applied fit to each data subset to compensate this dependency. In the 2-dimensional maps used in this study, the OFF-source datasets were summed over $3 \times 3$ adjacent subsets to obtain better statistics. Therefore, in this case, ratio of ON/OFF-source event counts is approximately 1 to 9 .

\section{Results}

\subsection{Two-dimensional significance map/latitude profile}

Fig. 3 shows contour plot of the resulting significance map (excess count divided by fitting error) for the $\ell=-19^{\circ} .5$ and $\ell=+13^{\circ} .0$

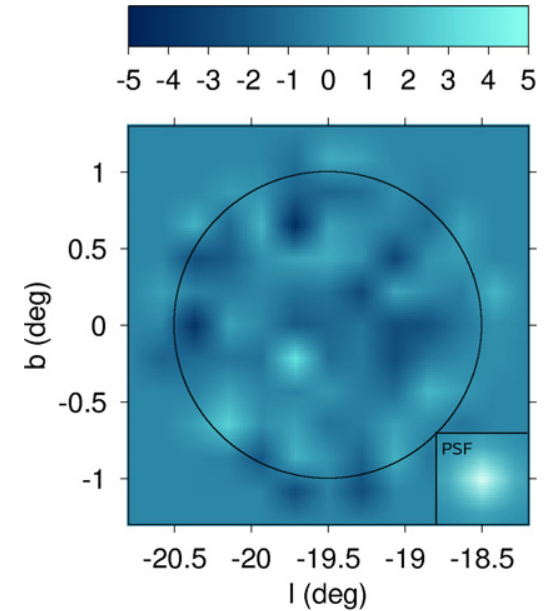

(a) $\ell=-19^{\circ} .5$

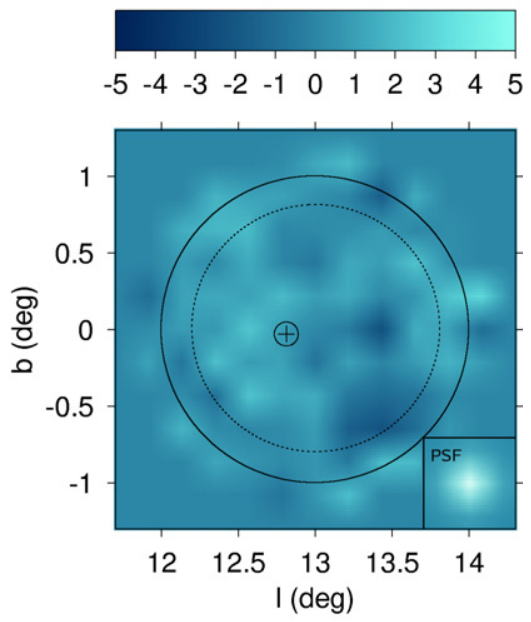

(b) $\ell=+13^{\circ} .0$

Fig. 3. Significance map of $(a)(\ell, b)=\left(-19^{\circ} \cdot 5,0^{\circ} .0\right)$ and $(b)(\ell, b)=\left(+13^{\circ} \cdot 0,0^{\circ} .0\right)$. The point spread function is also shown in the figures. Structures smaller than this PSF is likely due to statistical fluctuation. The cross on the right figure indicates the position of HESS J1813-178, which was in our field of view. The solid lines correspond to the area used in the analysis and the broken lines show the integration areas used in the derivation of the diffuse emission component flux. Size of these integration areas were determined by the $\mathrm{GeV}$ emission extent obtained by EGRET. As for $\ell=-19^{\circ} .5$ emission extent is larger $\left(1.1^{\circ}\right)$ than $1.0^{\circ}$, and we set integration area same as the solid line area.

regions. We selected a $0^{\circ} .2 \times 0^{\circ} .2$ square bin in the Galactic coordinate. The point spread function is also shown in the figures, for comparison with the size of statistical fluctuation. Only those events whose arrival direction were reconstructed within a $1^{\circ} .0$ radius from the center of the field of view were analyzed and displayed in the figures, because the gamma-ray acceptance is reduced by nearly one half at this radius. HESS J1813-178, a TeV point source, was located in the field of view for $\ell=+13^{\circ} .0$ and its position is denoted by a cross in the figure; however this source was not clearly identified. The consistency of our measurement with the H.E.S.S. result is discussed later.

Fig. 4 shows the latitude profile of flux intensity obtained by projection for the same regions as Fig. 3. In order to test the contribution of diffuse emission component, a circular region which corresponds to $\theta^{2}<0^{\circ} .1^{2}$ from a point source H.E.S.S. J1813-178 was eliminated from the analysis. The acceptance variance of the field of view and the difference in the projection areas for each bin was also corrected in the figures. 


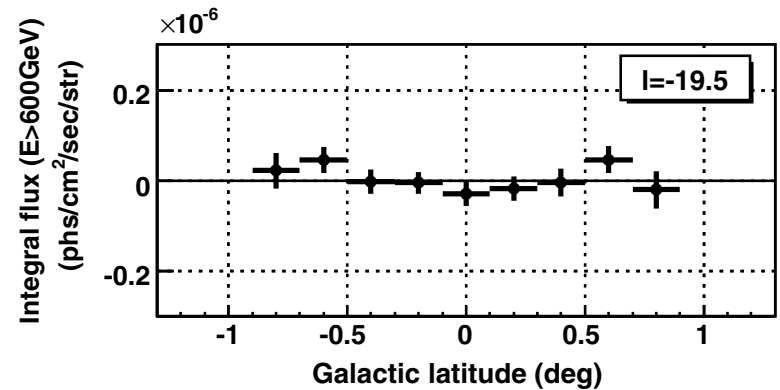

(a) $\ell=-19^{\circ} .5$

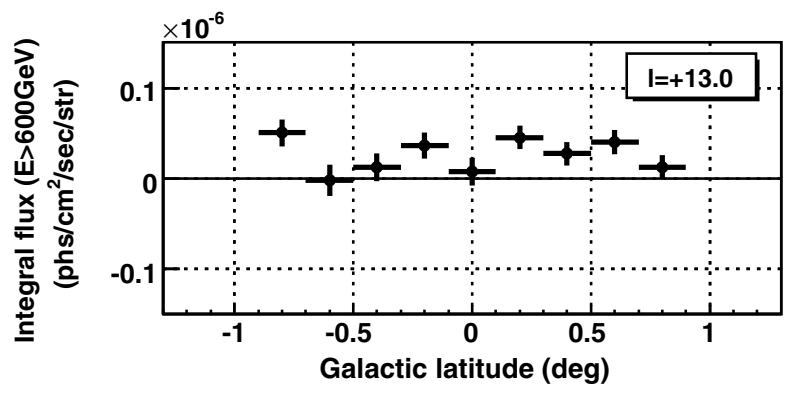

(b) $\ell=+13^{\circ} .0$

Fig. 4. The latitude profiles of the flux intensity for $\ell=-19^{\circ} .5$ and $\ell=+13^{\circ} .0$, obtained by the projection of 2-dimensional excess count map for the solid line disk region in Fig. 3. The raw count profile was divided by the acceptance and the solid angles for each projection bin. The bin-size in the latitude direction was selected to be $0.2^{\circ}$. Vertical bars represent fitting error in each data subset.

We did not observe any significant signal excess associated with the Galactic Plane at $b=0^{\circ} .0$ in either of the figures. We defined integration regions as disks whose radii are $1^{\circ} .0$ and $0^{\circ} .8$ for $\ell=-19^{\circ} .5$ and $\ell=+13^{\circ} .0$. The number of excess counts and their significance obtained by the CANGAROO-III observation are summarized in Table 3. We also applied a cut in Size (sum of the photo electron of an event) and checked the behavior of the result in the higher energy region. Our Size cut here was $>100$, which corresponds to $E>900 \mathrm{GeV}$.

For the $\ell=+13^{\circ} .0$ region, there seems to be a very weak and flat excess ( $\sim 3.7$ counts per $0^{\circ} .2 \times 0^{\circ} .2$ square bin) component over the field of view and its statistical significance is $+3.1 \sigma$ for $E>600 \mathrm{GeV}$ events after the integration. However, as mentioned before, in the analysis of extended sources, obtained excess count is very sensitive to the uncertainty of background level. Such a flat excess component can be generated by a small background level deviation and integration over the large area will produce large excess counts. Thus it is better to evaluate the significance of this excess with taking systematic error into consideration. This reduces significance

Table 3

Excess counts and flux upper limits obtained by CANGAROO-III observation

\begin{tabular}{lllllll}
\hline & $E_{\text {th }}(\mathrm{GeV})$ & Excess count & Solid angle & $\sigma_{\text {stat }}$ & $\sigma_{\text {tot }}$ & U.L. \\
\hline$\ell=-19^{\circ} .5$ & 600 & $38 \pm 137$ & $5.17 \times 10^{-4}$ & +0.3 & +0.3 & $1.1 \times 10^{-7}$ \\
& 900 & $-102 \pm 61$ & $5.17 \times 10^{-4}$ & -1.7 & -1.5 & $3.4 \times 10^{-8}$ \\
$\ell=+13^{\circ} .0$ & 600 & $168 \pm 55$ & $9.57 \times 10^{-4}$ & +3.1 & +2.4 & $1.9 \times 10^{-7}$ \\
& 900 & $49 \pm 20$ & $9.57 \times 10^{-4}$ & +2.5 & +2.2 & $4.7 \times 10^{-8}$
\end{tabular}

A gamma-ray spectrum described by a single power-law with $\alpha=-2.4$ was assumed between $1 \mathrm{GeV}$ and $10 \mathrm{TeV}$. The upper limits were derived in the form of an integral flux and then converted into a differential flux using the above assumption on the spectrum. The upper limit values in the table are in units of phs/ $\mathrm{cm}^{2} / \mathrm{s} / \mathrm{TeV} / \mathrm{str}$, with a $99.9 \%$ confidence level, in which systematic error was considered. $\sigma_{\text {tot }}$ is defined as $\sigma_{\text {tot }} \equiv \sqrt{\sigma_{\text {stat. }}^{2}+\sigma_{\text {syst. }}^{2}}$. Energy thresholds of 600 and $900 \mathrm{GeV}$ correspond to a size $>25$ and a size $>100$ cut, respectively. level to $+2.4 \sigma$ (detail about estimation of the systematic error is described later in the text).

One of the possible causes of background level deviation is difference of optical brightness between ON/OFF-source region. The $\ell=+13^{\circ} .0$ region is very bright in the optical band and low energy events tend to suffer from NSB noise effect. We expect better reliability for $E>900 \mathrm{GeV}$ events, where statistical significance of the excess is $+2.5 \sigma$, thus cannot be regarded significant.

With these facts we cannot conclude this excess in $E>600 \mathrm{GeV}$ was from the real gamma-ray emission and so we can set an upper limit to the flux with the systematic error. Better statistics in higher energy region by deep observation will improve the situation.

\subsection{Upper Limit of the diffuse emission component}

We were able to derive flux upper limits of the diffuse gammaray emission with this measurement. For the $\ell=+13^{\circ} .0$ region, point source effects were eliminated, as mentioned previously. The resulting flux upper limits with a 99.9\% confidence level are shown in Table 3 in the form of the differential flux at 600 and $900 \mathrm{GeV}$. Fig. 5 shows a comparison of the CANGAROO-III flux upper limits and the EGRET measurement. Since spatial binning of the EGRET data is a $0^{\circ} .5 \times 0^{\circ} .5$ square, a $2^{\circ} .0 \times 2^{\circ} .0$ square area was selected to perform the integration required for the comparison, which includes TeV integration area. In Fig. 5, extrapolation lines from the EGRET data point are also shown for different values of the spectral index. Only one data point at $1-2 \mathrm{GeV}$ was used in this extrapolation. According to [4], these $E>1 \mathrm{GeV}$ data points might suffer from systematic error in acceptance estimation and since this error is likely to be dependent on the gamma-ray energy, using the 1-2 GeV data point seems to be better than using higher energy points.

By comparing this EGRET data extrapolation with the CANGAROO-III flux upper limits for various spectral index assumptions, we can obtain lower limits on the spectral indices. The relation between the EGRET extrapolation and the CANGAROO-III upper limits for various spectral index sets is shown in Fig. 6. Since proton/ gamma-ray separation efficiency tends to be better in the higher energy region, we used the flux upper limit value obtained from $E>900 \mathrm{GeV}$ events in this analysis. The obtained lower limits on the spectral index are 2.2 for both of the $\ell=-19^{\circ} .5$ and $\ell=+13^{\circ} .0$ regions.

\subsection{HESS J1813-178: Consistency with the other measurement}

HESS J1813-178 is one of the sources discovered in the H.E.S.S. Galactic Plane survey [18]. In the study, this source is reported to be rather compact (radius of $2.2 \mathrm{arcmin}$ ), and integral flux for $E>200 \mathrm{GeV}$ was measured to be $(14.1 \pm 1.1) \times 10^{-12} \mathrm{phs} / \mathrm{cm}^{-2} / \mathrm{s}$, with a spectral index of $-2.09 \pm 0.05$. This object is associated with a radio source SNR G12.82-0.02. The MAGIC group also observed this target and they reported the detection of gamma-ray emission with an energy threshold of $100 \mathrm{GeV}$ [31] and a flux that was consistent with the H.E.S.S. result.

We could not clearly identify this compact source in Fig. 3 by this observation and we calculated flux upper limit for $\theta^{2}<0.1$ region. Obtained $\theta^{2}$ plot and upper limits with a $99.9 \%$ confidence level were shown in Fig. 7. Our upper limit is consistent with H.E.S.S. measurements.

\subsection{Systematic errors}

Measurement using atmospheric Cherenkov telescopes inevitably suffers from some form of systematic errors. One of the factors that cause such uncertainty is atmospheric transmittance variance which in turn affects the shower event rate. Another factor is the 


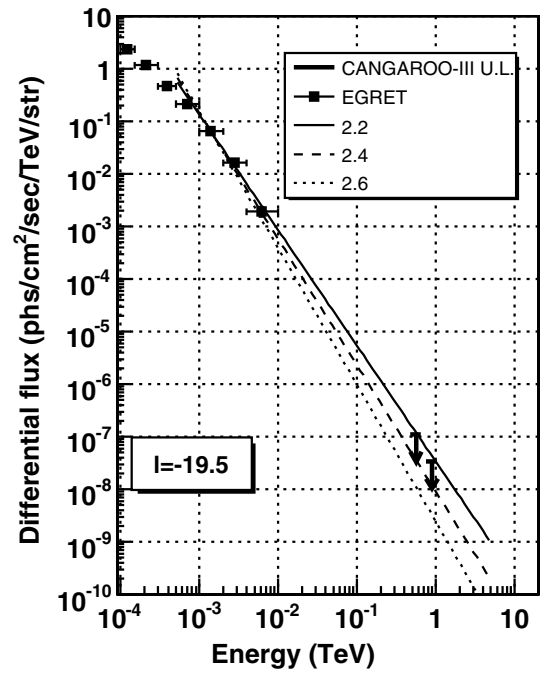

(a) $\ell=-19^{\circ} .5$

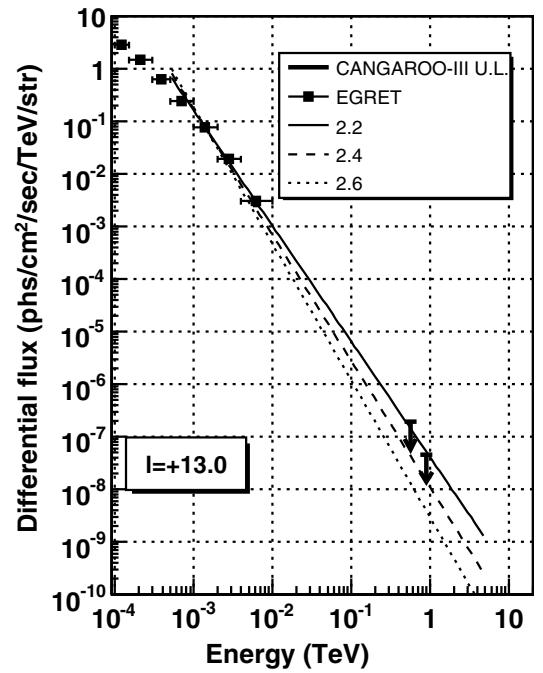

(b) $\ell=+13^{\circ} .0$

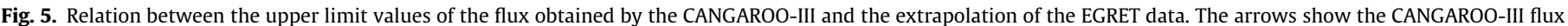
upper limit described in Table 3. The lines show the extrapolation of the EGRET 1-2 GeV data point, with some sets for different spectral index value.

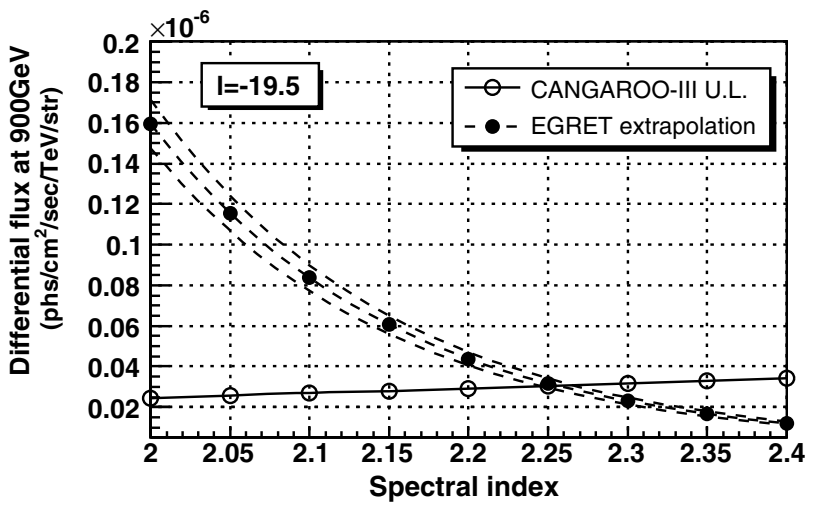

(a) $\ell=-19^{\circ} .5$

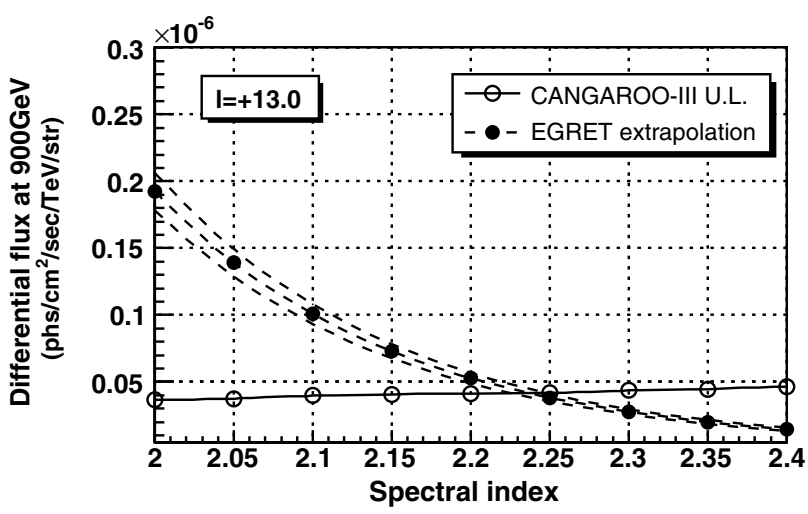

(b) $\ell=+13^{\circ} .0$

Fig. 6. Relation between the flux upper limits obtained by the CANGAROO-III and extrapolate from the EGRET data point for various spectral index values. Since the proton/gamma efficiency is better in the higher energy region, we use $E>900 \mathrm{GeV}$ events analysis in the figures. The broken lines indicate the extrapolation from an EGRET data point at $1-2 \mathrm{GeV}$ and the envelopes correspond to $1 \sigma$ statistical error. The solid lines indicate the flux upper limits obtained by CANGAROO-III. Given that the EGRET extrapolation should be lower than the CANGAROO-III flux upper limit, lower limits on the spectral indices were found to be 2.2 for both of the $\ell=-19^{\circ} .5$ and $\ell=+13^{\circ} .0$ regions.
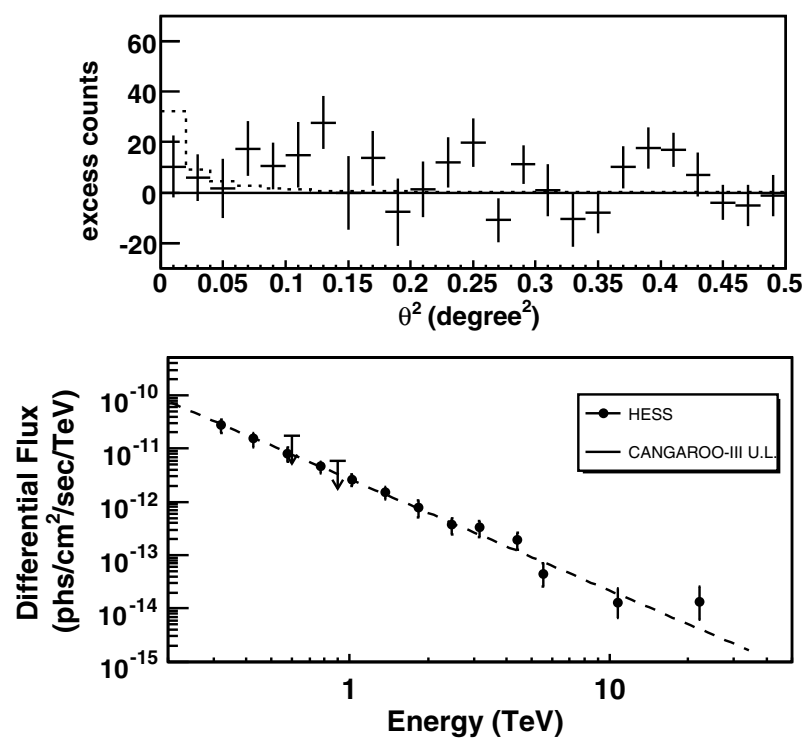

Fig. 7. $\theta^{2}$ plot of the J1813-178 (upper) and obtained flux upper limit (lower). The dashed line in the upper figure corresponds to the expected count estimated from the H.E.S.S. measurement. The upper limit values obtained by our observation are shown as arrows in the lower figure with a $99.9 \%$ confidence level.

uncertainty in the hardware parameter description used in the MC simulation. Although there are other sources of errors, we estimated the effect of these two factors on the upper limit values presented here.

The shower rate fluctuation is found to be $6 \%$ for $\ell=-19^{\circ} .5$ and $7 \%$ for $\ell=+13^{\circ} .0$ as the RMS of the day-averaged shower rate, both for $E>600 \mathrm{GeV}$ and $E>900 \mathrm{GeV}$. Regarding the Monte Carlo parameter uncertainty, we varied mirror reflectivity and optical spot-size within reasonable ranges in the simulation, and checked the fluctuation in the resulting upper limits. The magnitude of fluctuation expressed as an RMS is $23 \%$ for $\ell=-19^{\circ} .5$ and $26 \%$ for $\ell=+13^{\circ} .0(E>900 \mathrm{GeV})$. Combined with the shower rate fluctuation, it should be noted that the flux values we obtained include not less than $\sim 25 \%$ of systematic error. 


\section{Summary and discussion}

In 2004 the Galactic Plane was observed at two different longitude regions $\left(\ell=-19^{\circ} .5\right.$ and $\left.\ell=+13^{\circ} .0\right)$ with an energy threshold of $600 \mathrm{GeV}$ using the CANGAROO-III stereoscopic observation system. Events that triggered three telescopes were analyzed with an improved method incorporating both the Fisher Discriminant and a fitting technique. No significant signal associated with the Galactic Plane was observed from either of the regions. Assuming the gamma-ray spectrum described by a single power-law from a few $\mathrm{GeV}$ to $\mathrm{TeV}$, lower limits of the spectral indices for diffuse emission were found to be 2.2 for both of the $\ell=-19^{\circ} .5$ and $\ell=+13^{\circ} .0$ regions with a $99.9 \%$ confidence level. HESS J1813-178, a TeV point source associated with SNR G12.82-00.2, was in the field of view of the $\ell=+13^{\circ} .0$ region, although no significant excess was detected from this target by this observation. The upper limits imposed by our observation is consistent with the other measurements.

When we consider comparing our results with other observation results of the Galactic Plane, we conclude that the spectral index is a more suitable parameter than the flux which is sensitive to the local amount of target matter. H.E.S.S. revealed a very hard ( 2.3) gamma-ray spectrum for diffuse gamma-ray emission from the Galactic Plane near the Galactic Center [19], which is much harder than the locally measured proton spectrum (2.7). This means that the cosmic-ray proton spectrum is to some extent local; When the acceleration site exists nearby, the observed gamma-ray spectrum approaches the source spectrum. Even if that is the case it is not meaningless to compare spectral indices at different measurement points to see if the locally measured proton spectrum is a suitable Galactic average.

In this context, spectral indices (including lower limits) measured by past observations of the Galactic Plane and our results are summarized in Table 1 . With the present EGRET 1-2 GeV data point, our results are consistent with the other measurements.

Our lower limits on the spectral index are compatible with the EGRET measured spectrum of 2.45 extrapolated to the $\mathrm{TeV}$ region. Some authors have hypothesized a very hard electron spectrum (spectral index of $\sim 2.0$ ) inferred from radio observations of individual SNRs [5]. They deduced that the spatial distribution of electron spectrum is local, and the difference from the electron spectrum measured on the Earth can be explained by statistical fluctuation. This hard electron injection gives a gamma-ray spectral index of $\sim 1.85$; Our lower limits on the spectral index are larger than this value, therefore our results constrain the hypothesis of a very hard electron injection spectrum up to TeV level.

Deeper observations will give tighter constraint on the spectral index and test whether the EGRET data extrapolation is valid to $\mathrm{TeV}$ region and to clarify nature of the $\mathrm{GeV}$ anomaly. In addition to IACTs, satellite experiments will play an important role in such studies, by providing a large field of view. This study will be consigned to the next-generation gamma-ray satellite, such as GLAST [32].

\section{References}

[1] <http://cossc.gsfc.nasa.gov/docs/cgro/egret/>.

[2] D. Bertsch et al., ApJ 416 (1993) 587.

[3] S.D. Hunter et al., ApJ 481 (1997) 205

[4] F.W. Stecker, S.D. Hunter, D.A. Kniffen, Astropart. Phys. 29 (2008) 25.

[5] M. Pohl, J.A. Esposito, ApJ 507 (1998) 327.

[6] M. Mori, ApJ 478 (1997) 225.

[7] T. Kamae, T. Abe, T. Koi, ApJ 620 (2005) 244.

[8] A.W. Strong, I. Moskalenko, O. Reimer, ApJ 613 (2004) 962.

[9] T.C. Weekes et al., ApJ 342 (1989) 379.

[10] G. Vacanti et al., ApJ 377 (1991) 467.

[11] J. Hinton, in: Proceedings of 30th International Cosmic Ray Conference, in press.

[12] <http://vesper.icrr.u-tokyo.ac.jp>.

[13] <http://www.mpi-shd.mpg.de/hfm/HESS/HESS.html>

[14] <http://veritas.sao.arizona.edu>.

[15] <http://wwwmagic.mppmu.mpg.de>

[16] S. Lebohec et al., ApJ 539 (2000) 209.

[17] F.A. Aharonian et al., A\&A 375 (2001) 1008

[18] S. Funk et al., Science 307 (2005) 1938.

[19] F.A. Aharonian et al., Nature 439 (2006) 695.

[20] <http://umdgrb.umd.edu/cosmic/milagro.html>

[21] A. Abdo et al., ApJ 658 (2006) 33.

[22] A. Kawachi et al., Astropart. Phys. 14 (2001) 261.

[23] M. Ohishi et al., in: Proceedings of 28th International Cosmic Ray Conference, vol. 5, 2003, p. 2855.

[24] S. Kabuki et al., Nucl. Instrum. Meth. A 500 (2003) 318.

[25] H. Kubo, in: Proceedings of 28th International Cosmic Ray Conference, vol. 5, 2003, p. 2863.

[26] R. Enomoto et al., ApJ 638 (2006) 397.

[27] R. Enomoto et al., ApJ 652 (2006) 1268

[28] S. Kabuki et al., ApJ 668 (2007) 968.

[29] <ftp://legacy.gsfc.nasa.gov/compton/data/egret/>.

[30] D.J. Thompson et al., ApJS 86 (1993) 629.

[31] J. Albert et al., ApJL 637 (2006) 41.

[32] <http://glast.gsfc.nasa.gov/>.

[33] M. Amenomori et al., ApJ 580 (2002) 887.

[34] M. Amenomori et al., Science 314 (2006) 439. 